This item was submitted to Loughborough's Research Repository by the author.

Items in Figshare are protected by copyright, with all rights reserved, unless otherwise indicated.

\title{
Effective permittivity of heterogeneous substrates with cubes in a 3-D lattice
}

PLEASE CITE THE PUBLISHED VERSION

http://dx.doi.org/10.1109/LAWP.2011.2182597

PUBLISHER

(c) IEEE

VERSION

AM (Accepted Manuscript)

LICENCE

CC BY-NC-ND 4.0

REPOSITORY RECORD

Njoku, Chinwe C., W.G. Whittow, and J.C. Vardaxoglou. 2019. "Effective Permittivity of Heterogeneous Substrates with Cubes in a 3-D Lattice". figshare. https://hdl.handle.net/2134/9994. 
This item was submitted to Loughborough's Institutional Repository (https://dspace.lboro.ac.uk/) by the author and is made available under the following Creative Commons Licence conditions.

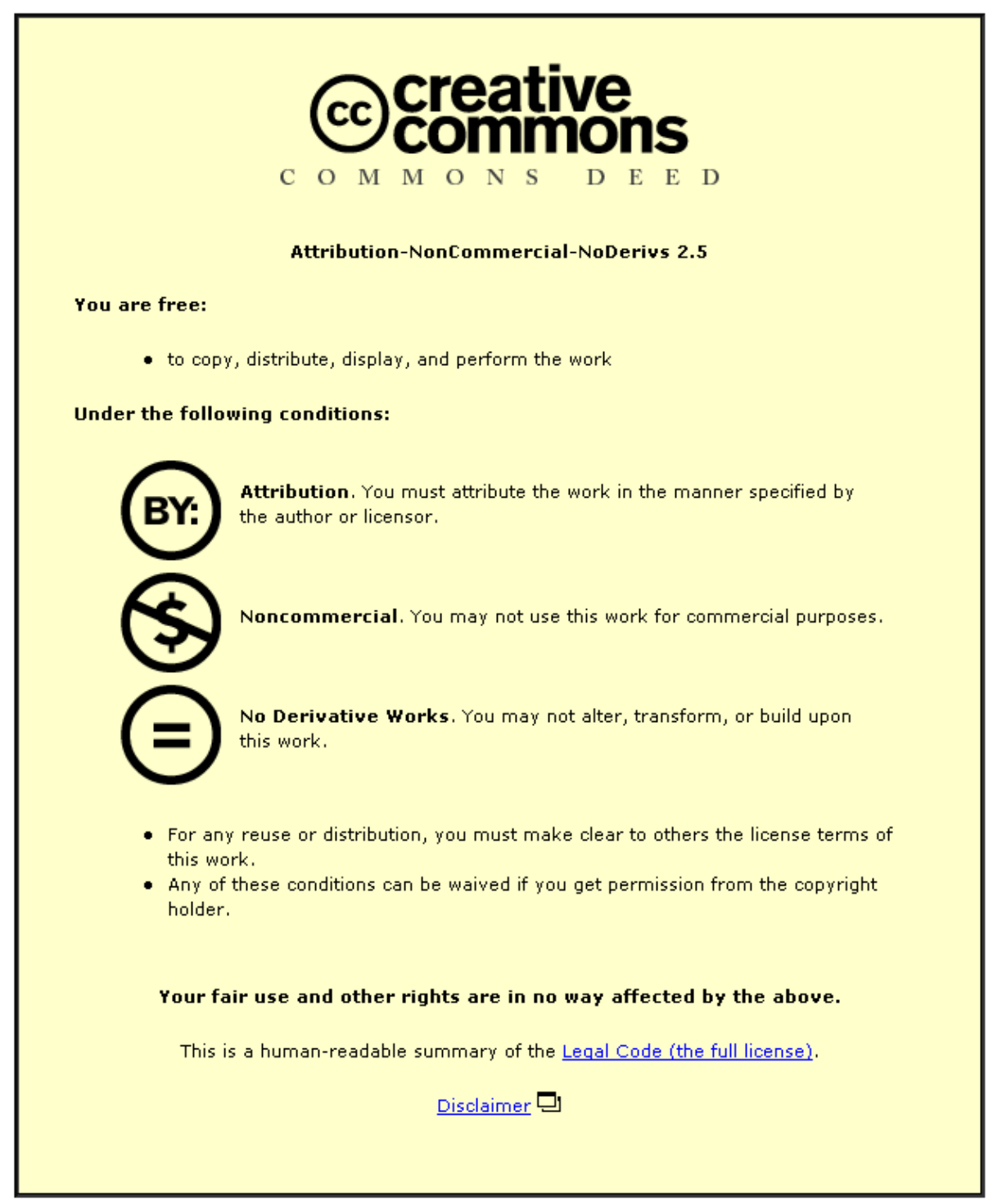

For the full text of this licence, please go to: http://creativecommons.org/licenses/by-nc-nd/2.5/ 


\title{
Effective Permittivity of Heterogeneous Sub- strates with Cubes in a 3-D Lattice
}

\author{
Chinwe C. Njoku, William G. Whittow, and J(Yiannis) C. Vardaxoglou, Senior Member, IEEE
}

\begin{abstract}
This letter studies the behaviour of heterogeneous dielectric substrates created by including micro-sized cubes of different electromagnetic (EM) properties to an otherwise homogenous host medium. The letter looks at the effect of the volume fraction of cubic inclusions (dielectric or metallic) to the effective EM properties of the heterogeneous medium. It is well known that the effective permittivity of the mixture is dependent on the properties of the host and inclusions as well as the size and spacing of the cubes. EM simulations using a plane wave excitation have been used to carry out these studies. An inversion process using a rectification algorithm was employed to correctly obtain the effective permittivity from the scattering parameters of the mixture. The analytical results of the infinite medium using canonical equations and a cube-sphere volume equivalence showed good agreement with the EM simulations and measurement of our samples of finite thickness. This letter shows that cubic inclusions can produce a higher effective permittivity than was previously found with spheres.
\end{abstract}

Index Terms-Cubic inclusions, effective permittivity, heterogeneous substrates.

\section{INTRODUCTION}

$\mathrm{O}$ $\mathrm{NE}$ of the most commonly used inclusion shapes in the creation of heterogeneous substrates is the sphere [1-6]. This is because the sphere is the same size in all three dimensions and as such is more straightforward to analyse as the induced field is uniform [2], [7], [8]. Other inclusion shapes include thin discs [9-11], cubes [8], ellipsoids [10], rectangular strips [11], I-shaped elements [12] amongst others. The use of other shapes which are non-uniform in all three dimensions, generally leads to anisotropy in which the EM properties of the medium may vary along different axes [10], [11], [13]. These heterogeneous composites allow the permittivity to be controlled and can be used as antenna substrates.

In creating heterogeneous substrates, the EM properties of the host material $\left(\varepsilon_{1}, \mu_{1}\right)$ and the inclusions $\left(\varepsilon_{2}, \mu_{2}\right)$ differ from each other. Depending on the size and spacing of the inclusions, and the uniformity of their arrangements, (e.g. in a

Manuscript received December 16, 2011; accepted December 19, 2011. This work was supported by the ESPRC under Grant No EP/I01490X/1.

The authors are with the Wireless Communications Research Group, School of Electronic, Electrical and Systems Engineering, Loughborough University, Loughborough LE11 3TU, U.K. (e-mail: j.c.vardaxoglou@lboro.ac.uk).

Color versions of one or more of the figures in this letter are available online at http://ieeexplore.ieee.org

Digital Object Identifier 10.1109/LAWP.2011.2182597 cubic lattice), the total mixture can be viewed on a macroscopic scale, as being homogenous; hence having an effective permittivity, $\varepsilon_{\text {eff }}$, and permeability, $\mu_{\text {eff }}$. This macroscopic behaviour can be obtained from the response of the inclusions or scatterers using [1], [2], and [8].

The aim of this letter is to investigate cubic inclusions and compare the results with previously published results with spherical inclusions. As shown in [14], a volume equivalence between spheres and cubes can be used to allow for comparison between spheres and cubes when using canonical equations given in [2]. A comprehensive analysis of these equations was done in [14-17]. This equivalence method has been shown to yield very good results. This letter shows that the multiplying factor limit on the host permittivity having metallic spherical inclusions can be significantly exceeded by the use of cubic inclusions in a cubic lattice.

Section II outlines the analysis of the various simulations carried out using the 3-D finite-difference time-domain (FDTD) software, EMPIRE XCcel. Results from canonical equations from [1], [2], and [6] using the sphere-cube volume equivalence approach were compared to the simulation results. Dielectric and metallic cubes of the same size but different spacings were used. In Section III, results from further EM analysis are discussed. Measurements were carried out to show the effectiveness of the theoretical study and are discussed in Section IV. Finally, conclusions are made in Section $\mathrm{V}$.

\section{Electromagnetic Simulations}

Canonical equations in the literature use spherical inclusions for their analysis. EM simulations in this letter used mixtures with cubic inclusions for the analysis. It is well known that the maximum packing density for spherical inclusions is when the spheres are touching, which implies a volume fraction of $\pi / 6 \approx 0.5$, for a simple cubic (SC) lattice arrangement. This theoretical barrier of the volume fraction limits the extent to which the effective permittivity of the host medium can be raised. Given that metallic particles have much higher equivalent permittivities [2], [18], it has been shown in [2] that the highest multiplying factor for metallic spherical particles in an SC lattice can have on the host material is $\sim 4.297$. This factor is irrespective of the size and spacing of the particles as the volume fraction has a dominating effect on the $\varepsilon_{\text {eff }}$ of the mixture. A factor of up to $\sim 8$ and 10 may be obtained with spherical inclusions in the body and face centred cubic lattices respectively, where the volume fraction is larger [3], [13]. 
VOL. 10, pp 1480-1483, 2011

This prompts the need to study other inclusion shapes to break these limits, thus the use of cubic inclusions here. As the cubes can occupy more of the volume space in their unit cells, $\varepsilon_{e f f}$ is higher. As the size of the cubes tends to that of the unit cells, it is expected that the $\varepsilon_{e f f}$ of the mixture would approach the permittivity of the inclusions. For a fixed cube size, reducing the spacing between them eventually leads to a situation where their equivalent spheres are overlapping which is physically unrealisable. Using cubes is therefore helpful in terms of maximising the volume fraction. Reference [8] examines the polarisability of dielectric cubes using a "surfaceintegral-equation" approach, in order to check that when used in the well-known Maxwell-Garnett equation for effective permittivity, a reasonably accurate result can be obtained [10].

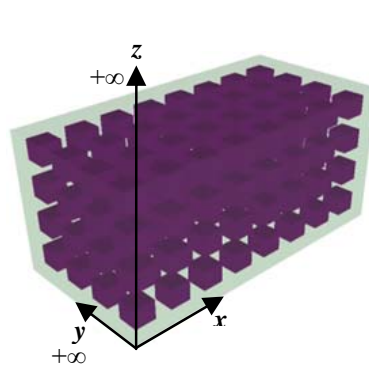

(a)

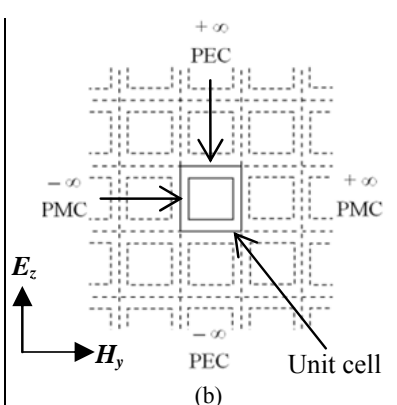

(b)

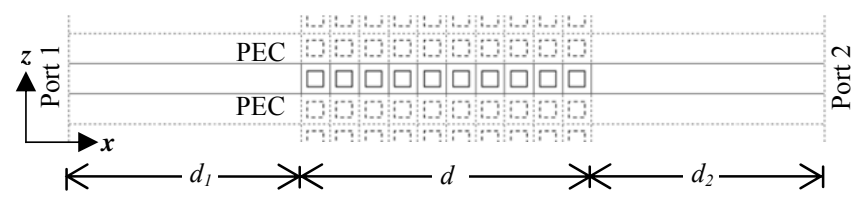

(c)

Fig. 1. (a) 3-D representation of a $4 \times 4$ section of the infinite array. (b), (c) 2$\mathrm{D}$ views of a 10-layer simulation setup of the heterogeneous structure. The dotted lines represent the planes of symmetry where the structure extends to infinity and are represented by using PECs and PMCs.

Fig. 1 shows the simulation arrangement of the cubic inclusions in a uniform lattice in three and two dimensions. The cubes are equally spaced in all three dimensions. The simulations are set up such that the boundary conditions in two dimensions $(y$ and $z$ ) are perfect electric and magnetic conductors (PECs and PMCs), as shown in Fig. 1(b), while in the third dimension $(x)$, perfectly matched layers (PMLs) are used to minimise reflections, see Fig. 1(c). The PEC and PMC boundaries create a structure that is infinite in two dimensions - parallel to the plane of incidence of the plane wave. The structure is however, finite (thickness $d$ ) in the direction of propagation of the EM wave.

A Resonant Inverse Scattering formalism together with a rectification algorithm [9] is used to extract the effective EM properties of the heterogeneous mixture, further details of which can be found in [14]. This method uses the scattering (S-) parameters and thickness of the medium when subjected to a plane wave excitation to obtain these properties. The Sparameters measured at the port locations are first subject to phase corrections to account for the shift from the edge of the structure to the port positions [19]. The rectification algorithm corrects the phase of the medium as it may exhibit sharp discontinuities over the frequency range.

Table I summarises the symbols used for the parameters. The permittivity values used in this letter are magnitudes of their relative values.

TABLE I

LIST OF SYMBOLS FOR PARAMETERS USED

\begin{tabular}{|c|l|}
\hline \hline$l$ & Length of cubic inclusions \\
\hline$s$ & Centre-to-centre spacing of inclusions \\
\hline$p$ & Volume fraction of inclusions \\
\hline$d$ & Sample thickness \\
\hline$\varepsilon_{1}, \mu_{1}$ & Host permittivity and permeability \\
\hline$\varepsilon_{2}, \mu_{2}$ & Inclusion's permittivity and permeability \\
\hline$\varepsilon_{e f f}, \mu_{e f f}$ & Effective permittivity and permeability \\
\hline
\end{tabular}

\section{A. Dielectric Cubic Inclusions}

As a first step in the analysis of cubic inclusions, the host and the inclusions were both dielectric. Data used: $l=100 \mu \mathrm{m}$, $s=105-250 \mu \mathrm{m}, \varepsilon_{1}=2.25, \varepsilon_{2}=11.9, \mu_{1}=\mu_{2}=1$. Fig. 2 shows the variation of $\varepsilon_{e f f}$ with frequency for different inclusion spacings, while Fig. 3 shows the decrease in $\varepsilon_{\text {eff }}$ with increase in spacing at $10 \mathrm{GHz}$.

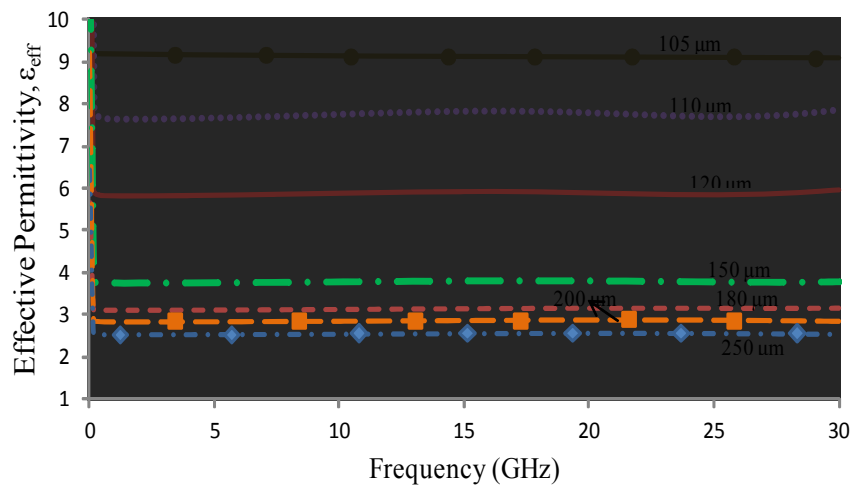

Fig. 2. Variation of $\varepsilon_{e f f}$ with frequency for $100 \mu \mathrm{m}$ dielectric cubes spaced $105 \mu \mathrm{m}($ (-•), $110 \mu \mathrm{m}(\cdots \bullet \bullet), 120 \mu \mathrm{m}(-), 150 \mu \mathrm{m}(-\bullet-), 180 \mu \mathrm{m}$ $(-), 200 \mu \mathrm{m}(--)$ and $250 \mu \mathrm{m}(-\bullet-)$ ) apart.

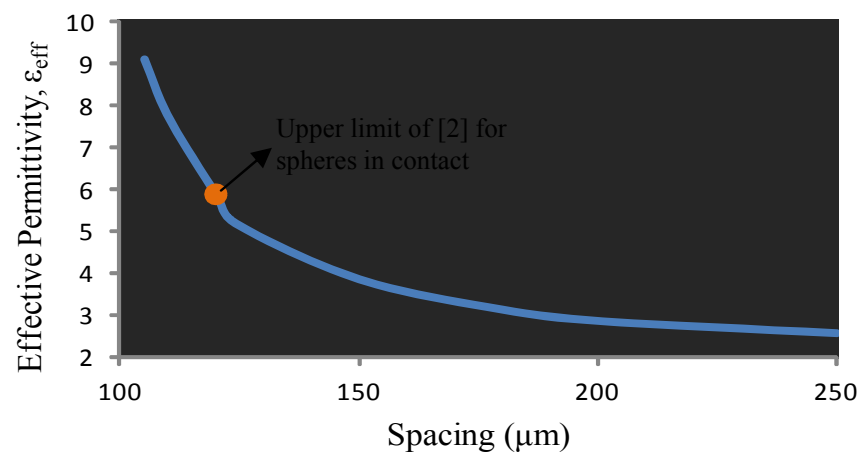

Fig. 3. Variation of $\varepsilon_{e f f}$ with spacing at $10 \mathrm{GHz}$.

As shown in Fig. 2, there is a 1.8 difference in the $\varepsilon_{\text {eff }}$ value when the spacing is reduced by $10 \mu \mathrm{m}$ from $120 \mu \mathrm{m}$ to 110 
$\mu \mathrm{m}$. The marker in Fig. 3 shows the $\varepsilon_{\text {eff }}$ for when the cubes replaced by volume-equivalent spheres are touching in an SC lattice. Note, if the spheres were touching, the $\varepsilon_{\text {eff }}$ would increase to $\sim 2.34$ times the host. This value could be increased towards the 4.297 limit by increasing the $\varepsilon_{2}$ of the inclusions. From [8], formulas for the polarisability of a cube were derived. When used in the Clausius-Mossotti equation for effective permittivity [10], with $l=100 \mu \mathrm{m}, s=150 \mu \mathrm{m}, \varepsilon_{1}=$ $2.25(\tan \delta=0.001), \varepsilon_{2}=11.9(\tan \delta=0.01), \varepsilon_{\text {eff }}$ was obtained as $3.798-\mathrm{j} 0.014$, which is in good agreement with that obtained from the simulations.

\section{B. Metallic Cubic Inclusions}

The Drude model produces an equivalent permittivity of metal which is typically much greater than that of dielectrics [18]. Therefore, metallic inclusions are expected to have a larger effect on the overall permittivity of the mixtures. With metallic spheres, the highest $\varepsilon_{\text {eff }}$ theoretically possible in an SC lattice is about four times that of the host [2]. It was expected that with cubic inclusions this limit should be broken. Data used: $l=100 \mu \mathrm{m}, s=105-250 \mu \mathrm{m}, \varepsilon_{1}=2.25(\tan \delta=$ $0.001)$, inclusion material $=$ silver, $\left(\sigma=6.1 \times 10^{7} \mathrm{~S} / \mathrm{m}\right)$, $\mu_{1}=\mu_{2}=1$. Fig. 4 shows the different $\varepsilon_{\text {eff }}$ values for different spacings.

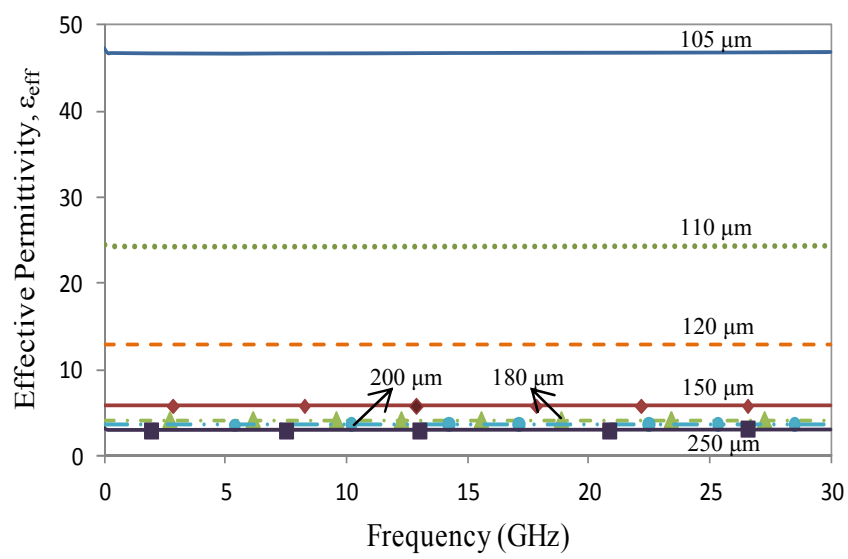

Fig. 4. Variation of $\varepsilon_{\text {eff }}$ with frequency for $100 \mu \mathrm{m}$ metallic cubes spaced $105 \mu \mathrm{m}(-), 110 \mu \mathrm{m}(\bullet \bullet), 120 \mu \mathrm{m}(---), 150 \mu \mathrm{m}(-\bullet), 180 \mu \mathrm{m}\left(-\cdot \boldsymbol{A}^{-}-\right.$ ), $200 \mu \mathrm{m}(-\cdots \cdots)$ and $250 \mu \mathrm{m}(-\square-)$ apart.

As shown in Fig. 4, when the cubes are $5 \mu \mathrm{m}$ apart, an $\varepsilon_{\text {eff }}$ of 46.7 is obtained, an increase of 21.2 times. It is expected that even higher $\varepsilon_{\text {eff }}$ could be obtained by further reducing the spacing. However, in the limit where the metallic cubes are touching, the object will behave like a continuous metal structure. As expected, metallic cubic inclusions produce a significantly larger $\varepsilon_{\text {eff }}$ than either metallic spherical inclusions or dielectric cubic inclusions, considered in Fig. 2.

\section{FURTHER ANALYSIS AND RESUltS}

In order to understand the behaviour of the metallic cubes within the dielectric medium, further simulations and analysis

\section{were carried out.}

\section{A. Loss Tangents}

The loss tangent values of the $\varepsilon_{\text {eff }}$ were calculated using $\tan \delta=\operatorname{imag}\left(\varepsilon_{e f f}\right) / \operatorname{real}\left(\varepsilon_{e f f}\right)$ for different spacing-size ratios with the 100-, 75- and 40- $\mu \mathrm{m}$ metallic cubes, at $10 \mathrm{GHz}$. This gave an average loss tangent value of 0.001 .

Even when the cubes were in very close proximity, the heterogeneous structure has very low loss and approximates the $\tan \delta$ of the host. These low loss tangent values may be due to the low loss tangent value of the host medium and the high conductivity value of the inclusions.

\section{B. Spacing-to-size Ratios}

Further simulations were carried out using the same spacing-to-size ratios (equivalent to volume fractions) while changing the size of the metallic cubes. The cube sizes used were 100,75 and $40 \mu \mathrm{m}$, with the same EM values as in Section IIB. Fig. 5 shows the variation of $\varepsilon_{\text {eff }}$ for each cube size with this ratio.

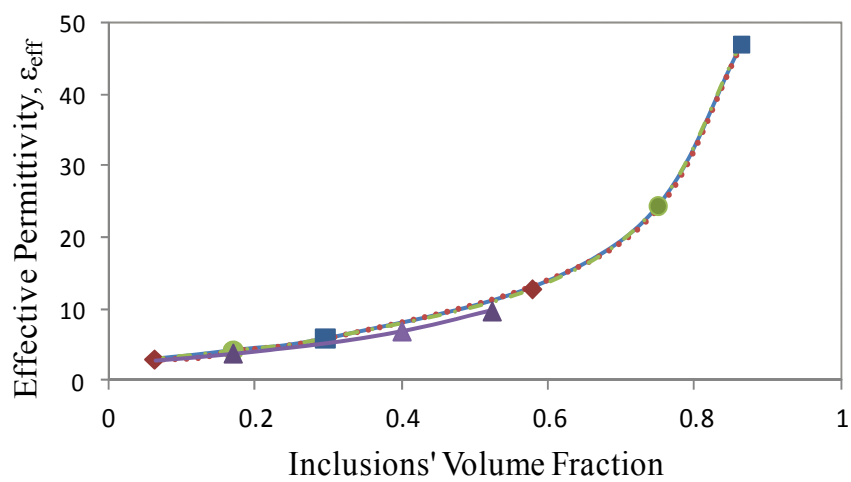

Fig. 5. Variation of $\varepsilon_{\text {eff }}$ with inclusions' volume fraction from simulations for $100 \mu \mathrm{m}(-\bullet-), 75 \mu \mathrm{m}(\bullet \bullet \bullet \bullet)$, and $40 \mu \mathrm{m}(-\bullet \bullet-)$ cubes at $10 \mathrm{GHz}$; and from canonical equations $(-\boldsymbol{\Delta}-)$ [1], [2], [6].

As shown in Fig. 5, the canonical results using [1], [2], and [6], (which all give similar results) calculated with the volume equivalence method, show very good agreement with the results from the simulations. Note, [1], [2], and [6] are for spheres and only allow up to a 0.524 volume fraction. It can be concluded that as in the case of the spherical inclusions, the volume fraction of the inclusions play a dominant role in determining the $\varepsilon_{\text {eff } f}$ of the medium. The size of the cubes plays little role in determining the overall permittivity of the structure. This could mean that as long as the frequency of operation is well below the resonant frequency of the inclusions, the same $\varepsilon_{e f f}$ can be predicted even when the particles' size is reduced.

\section{RESUlts From MEASUREMENTS}

Initial scaled-up measurements using spherical metallic inclusions in a Rohacell ${ }^{\circledR}$ host were carried and the results discussed in [14]. As the structures examined in this letter are cubical, copper $(\mathrm{Cu})$ squares of identical sizes and periodicity 
(along the axes of the squares only) were etched on both sides of a GTS substrate with a relative permittivity of 3.0. Data used: $\mathrm{Cu}$ square size $=500 \times 500 \times 35 \mu \mathrm{m}^{3}$, periodicity $=750$ $\mu \mathrm{m}$, frequency range $=1-6 \mathrm{GHz}$. A three-layer sample of volume fraction, 0.173 and thickness $540 \mu \mathrm{m}$, was assembled and measured using the resonator technique in [20]. In our case we used a half-wavelength resonator of length $19 \mathrm{~mm}$, which was printed on a 1.125-mm-thick layer of Taconic. The resonator was printed on a $140-\mu \mathrm{m}$-thick GTS layer. We have validated this technique by measuring accurately samples of known dielectric constants. Fig. 6 shows the measurement set-up.

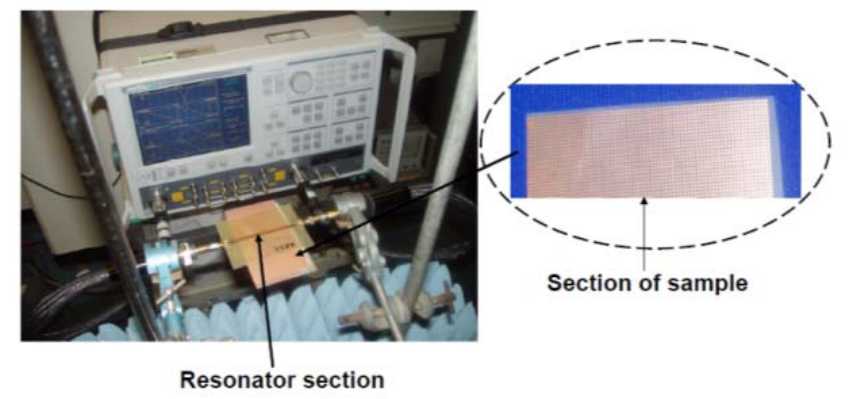

Fig. 6. Measurement setup

The effective permittivity of the sample in the above measurement setup was estimated from the well-known equations used in [20] and from [21, Eq. (13)]. The values obtained were in good agreement: 6.52 from simulations and 6.34 from the measurements.

\section{CONCLUSION}

It has been shown that the restriction on the maximum possible value of effective permittivity with the use of spheres can be significantly reduced by the use of cubic inclusions. A multiplying factor, approximately 20 times the permittivity of the host has been achieved with the use of cubic metallic inclusions. Further enhancements may be possible with more closely packed cubes. The effective loss tangents from these heterogeneous media obtained from simulations are similar to the loss tangent of the host medium. This letter has shown that the inclusion material and volume fraction are the key parameters. Therefore, these results are independent of scale, assuming the inclusion sizes are much less than the operating wavelengths. Measurement result of a 3-layer sample in a resonator arrangement was in good agreement with the simulated value.

\section{ACKNOWLEDGEMENT}

The authors wish to thank Dr. A. Chauraya for carrying out the measurements.

\section{REFERENCES}

[1] W. T. Doyle, "Optical properties of a suspension of metal spheres", Phy. Rev. B, vol. 39, issue 14, pp. 9852-9858, May 1989.

[2] L. Lewin, "The electrical constants of a material loaded with spherical particles", IEE-Part III: Radio Commun. Eng., vol. 94, no. 27, pp. 6568, Jan. 1947.
[3] I. Awai, O. Mizue, and A. K. Saha, "Artificial dielectric resonator made of spherical metal particles," Inst. Elect. Infor. Commun. Eng., vol. E92C, no. 1, pp. 72-76, 2009.

[4] X. Cai, R. Zhu, and G. Hu, "Experimental study for metamaterials based on dielectric resonators and wire frame", Metamaterials, vol. 2, no. 4, pp. 220-226, Sept. 2008.

[5] L. Rayleigh, "On the influence of obstacles arranged in rectangular order on the properties of a medium", Phil. Mag., ser. 5, vol. 34, pp. 481-502, 1892 .

[6] I. A. Kolmakov, L. Jylha, S. A. Tretyakov, and S. Maslovki "Lattice of dielectric particles with double negative response", presented at $28^{\text {th }}$ Gen. Assembly URSI, 2005, Paper BCD.5 (0109).pdf.

[7] J. A. Stratton, Electromagnetic Theory. New York: McGraw-Hill, 1941, ch. 9.

[8] J. Avelin, A. Sihvola, R. Sharma, and I. Hanninen, "Modelling of dielectric materials with cubic inclusion shapes," in Proc. 29th Eur. Microw. Conf., Mar. 2007, pp. 36-39.

[9] N. G. Alexopoulos, C. A. Kyriazidou, and H. F. Contopanagos, "Effective parameters for metamorphic materials and metamaterials through a resonant inverse scattering approach", IEEE Trans. Microw. Theory Tech., vol. 55, no. 2, pp. 254-267, Feb. 2007.

[10] A. Sihvola, Electromagnetic Mixing Formulas and Applications, ser. Elect. Waves 47. London, U.K.: IEE, 1999, ch. 3.

[11] R. E. Collin, Field Theory of Guided Waves. New York: IEEE Press, 1991 , ch. 12

[12] L. Xing, Q. Xu, J. Li, Z. Wei, J. Ding, and C. Guo, "Broaden the bandwidth of patch antenna by using inhomogeneous metamaterial substrate," in Proc. Prog. Electromagn. Res. Symp., 2010, vol. 1, pp. 155159.

[13] I. Awai, Y. Maegawa, and T. Ishizaki, "Measurement of effective material constants of artificial dielectrics made of spherical metal particles", in Proc. APMC 2009, Jan. 2010, pp. 1655-1658.

[14] C. C. Njoku, W. G. Whittow, and Y. C. Vardaxoglou, "Simulation methodology for synthesis of antenna substrates with micro-scale inclusions," IEEE Trans. Antennas Propag., 2011, to be published.

[15] C. C. Njoku, W. G. Whittow, and Y. C. Vardaxoglou, "Comparative study of nanomaterials' effective properties using canonical equations," in Proc. 7th Annu. LAPC, Loughborough, U.K., 2010, pp. 413-416.

[16] C. C. Njoku, W. G. Whittow, and Y. C. Vardaxoglou, "Microwave antennas and heterogeneous substrates using nanomaterial fabrication techniques," in Proc. IEEE APWC, Turin, Italy, Sep. 12-17, 2011, pp. 843-846.

[17] C. C. Njoku, W. G. Whittow, and Y. C. Vardaxoglou, "Study on the variation in dielectric properties of heterogeneous substrates composed of nanomaterials," in Proc. 5th EuCAP, 2011, pp. 488-492.

[18] M. A. Ordal, et al., "Optical properties of the metals $\mathrm{Al}, \mathrm{Co}, \mathrm{Cu}, \mathrm{Au}, \mathrm{Fe}$, $\mathrm{Pb}, \mathrm{Ni}, \mathrm{Pd}, \mathrm{Pt}, \mathrm{Ag}, \mathrm{Ti}$, and $\mathrm{W}$ in the infrared and far infrared", Appl. Opt., vol. 22, no. 7, Apr. 1983, pp. 1099-1119.

[19] W. B. Weir, "Automatic measurement of complex dielectric constant and permeability at microwave frequencies", Proc. IEEE, vol. 62, no. 1, pp. 33-36, Jan. 1974

[20] P. A. Bernard, and J. M. Gautray, "Measurement of dielectric constant using a microstrip ring resonator," IEEE Trans. Microw. Theory Tech., vol. 39, no. 3, pp. 592-595, Mar. 1991.

[21] F.-G. Hu, J. Song, and T. Kamagaing, "Modeling of multilayered media using effective medium theory," in Proc. $19^{\text {th }}$ Annu. Conf. EPEPS, Oct. 25-27, 2010, pp. 225-228. 